\title{
A Rarely Seen Pathology "Intramedullary Spinal Metastasis": Clinical Series of Five Patients in a Single Institution
}

\author{
Yener Akyuva' (1), Neşe Karadağ² (1), Çağatay Önal ${ }^{3}$ (1) \\ 1Department of Neurosurgery, University of Health Sciences Gaziosmanpaşa Taksim Training and Research Hospital, İstanbul, Turkey \\ ${ }^{2}$ Department of Pathology, Inönü University School of Medicine, Malatya, Turkey \\ ${ }^{3}$ Department of Neurosurgery, Inönü University School of Medicine, Malatya, Turkey
}

Cite this article as: Akyuva Y, Karadağ N, Önal Ç. A Rarely Seen Pathology "Intramedullary Spinal Metastasis": Clinical Series of Five Patients in a Single Institution. JAREM 2018; 8(3): 138-46.

\begin{abstract}
Objective: Our study is related to our experiences with intramedullary spinal cord metastasis (ISCM). The purpose of the present study was to evaluate the clinical features, treatment, and natural course of patients in the context of the literature.

Methods: Five patients with ISCM who were admitted to the neurosurgery department between October 2011 and December 2016 and who underwent surgery were identified. Relevant clinical data were obtained.

Results: Of the five patients, three had lung cancer, one had breast cancer, and one had renal cell carcinoma. The presenting symptoms were pain, urinary incontinence, and/or weakness. Tumors were at the thoracic level in three patients, cervical level in one patient, and thoracolumbar level in one patient. One patient with lung cancer had undergone metastasectomy for intracranial metastasis. The pathological examination of one patient had been reported as anaplastic ependymoma in a previous health facility, but breast cancer metastasis was found to be the primary diagnosis following the examination of the material obtained from the excision of the relapsed tumor.

Conclusion: The diagnosis of ISCM is difficult, and treatment is usually ineffective. Although there is no exact treatment modality in ISCM, appropriate surgery positively affects morbidity and mortality. Planning the right treatment for the right patient is the most important step of ISCM management.

Keywords: Intramedullary tumors, spinal cord, spinal cord metastases, spinal cord surgery
\end{abstract}

ORCID IDs of the authors: Y.A. 0000-0001-8171-5929; N.K. 0000-0002-6303-5484; Ç.Ö. 0000-0002-1216-2301.

\section{INTRODUCTION}

Intramedullary spinal cord metastases (ISCMs) are parenchymal metastases of the spinal cord and are rare as central nervous system metastases of the malignant tumors. They are present in $0.1 \%-0.4 \%$ of all patients with cancer and constitute $1 \%-3 \%$ of intramedullary spinal cord tumors (1). In many patients, other systemic metastases are present at the time of diagnosis (2).

Metastases are most frequently seen in the lumbar and thoracic regions and less frequently in the cervical region $(3,4)$. They are often seen as a single lesion. Brain metastases are present in the majority (35\%) of patients, and, to a lesser extent, leptomeningeal carcinomatosis may be present $(2,5)$. Lung cancer is the most common (54\%) ISCM-causing tumor, and small cell lung cancer is the most common subtype. Other than this, ISCMs may be seen in breast cancer (13\%), renal cancer (4\%), melanomas (9\%), and/ or lymphomas (5\%) (6-10).
In metastatic spinal tumors, the age of diagnosis is between 50 and 60 years. It is reported that males are more frequently affected than females (11). It is reported that symptoms occur in 2 months on average, the first complaint is pain and myelopathy, and plegia may develop in $10 \%-15 \%$ of cases (3). More than half of the cases have motor deficits, and approximately half of them have sphincter problems and sensory disturbances $(9,12)$.

Magnetic resonance image (MRI) plays an important role in surgical planning when ISCM is suspected in the differential diagnosis. In some cases, computed tomography (CT) and CT myelography are helpful in the diagnosis and treatment $(3,11)$. ISCMs have high mortality and morbidity rates. Parallel to the development of MRI techniques, differential diagnosis rates are increasing. However, with improved microsurgical and oncological treatment modalities, better survival and local control rates have been achieved in treated cases.

In ISCM cases, if possible, primary treatment is decompression through microsurgical tumor excision. In this way, tumor burden 
can be reduced, and an area where the neural parenchyma can be expanded can be gained. In cases where the primary tumor is uncertain, information about the primary focus can be obtained by excision; in addition, adjuvant systemic chemotherapy (CTX) or local radiotherapy (RT) can be added to surgery.

In cases where primary disease is known or tissue is diagnosed by excision, RT conformal, radiosurgery (SRS), or stereotactic body RT (SBRT), which has been used more frequently recently as an adjuvant to surgery, is used to increase local control. Systemic CTX regimes can be determined according to primary disease histopathology.

Although there is a multidisciplinary approach in ISCM management, there is no clear optimal treatment modality. In most patients, tumor recurrence develops despite surgical treatment, RT, such as SRS and/or SBRT treatment modalities, or CTX. In addition, surgery for recurrent mass excision may be necessary. In such cases, the most effective treatment is the application of treatment modalities, which include effective metastasectomy, in the shortest time.

The aim of the present study was to compare the findings of patients with ISCM who underwent microsurgery and adjuvant RT with data obtained from the systematic evaluation of clinical studies in the literature.

\section{METHODS}

The present study was performed according to the framework of the Declaration of Helsinki. Written consent was obtained from the patients.

\section{Eligibility Criteria for Patients}

The study population comprised patients $(n=242)$ who were admitted to the neurosurgery outpatient clinic between October 2011 and December 2016 with complaints of numbness in the extremities, loss of strength, and stool and/or urinary incontinence. Patients who were included in the present study $(n=5)$ had been diagnosed histopathologically as having ISCM following microsurgical intramedullary tumor excision.

\section{Surgical Technique}

The surgery included bilateral total laminectomy at the tumor level, dural opening from the posterior, dorsal midline myelotomy, and microsurgical tumor excision, followed by decompression surgery with extensive duraplasty with fascial graft.

\section{Search Strategy and Selection Criteria}

The Cochrane Collaboration, Cochrane Library (Issue 2, 2/12/2011), Ovid MEDLINE (1950 to 10/31/2016), ProQuest, US National Library of Medicine National Institutes of Health, and PubMed electronic databases were searched for clinical studies using the keywords "intramedullary spinal cord metastasis", "carcinoma metastasis", "renal cell carcinoma", "breast cancer", "intracranial metastasis", "lung adenocarcinoma", and "lung cancer" without any language or country limitation.

Of all the retrieved studies, those with high levels of evidence were selected. The study by Lijmer et al. (13) was used to determine the level of evidence of research. Randomized, controlled and double-blind studies with high levels of evidence were identified. Publications that did not meet all of these criteria were excluded from the study. All bibliographies considered to have been missed during the database search were re-evaluated. The reference lists were also evaluated in terms of the availability of appropriate articles. However, unpublished studies, comments, letters to editors, editorials, protocols, guidelines, meta-analysis, and reviews were excluded from the study.

Later, the most cited studies were identified. The references and citations of these studies were examined to avoid possible repetition. After full-text evaluation, they were arranged according to the year of publication. Finally, a total of 16 research articles, including the aforementioned search terms, were included in the present study $(10,14-29)$.

\section{Data Acquisition and Evaluation}

The studies included in the present study were selected independently. The risk of selection bias due to potential masking was also explored. All studies were examined by three authors to ensure correctness. In case of disagreement between the three authors, a consensus was reached through the facilitation of the senior author.

\section{Statistical Analysis}

It was found that the obtained data were not based upon the fact that they had been collected from sources with probability distribution function. Therefore, non-pragmatic statistical methods were used. However, given the lack of common findings, statistical analyses could not be performed, and, instead, complementary statistical methods were applied. Microsoft Office Excel 2010 was used for statistical analysis. Results were expressed as mean \pm standard deviation or frequency (\%).

\section{RESULTS}

Between October 2011 and December 2016, 242 patients who were admitted to the neurosurgery outpatient clinic with complaints of numbness in the extremities, loss of strength, and stool and/or urinary incontinence were detected. Among these patients, five had a histopathological diagnosis of ISCM after tumor excision (Figure 1).

It was found that three patients had lung cancer, one had breast cancer, and one had renal cell carcinoma (RCC) diagnosis. In addition, it was determined that two patients had not been diagnosed with cancer prior to surgery, and that they were diagnosed postoperatively as having ISCM due to lung cancer. It was observed that one patient with lung cancer had undergone metastasectomy for intracranial metastasis in the same clinic (Table 1).

In addition to MRI and CT, it was observed that excision materials were histopathologically evaluated after routine tissue processing for differential diagnosis. Immunohistochemical studies, such as hormone receptors in breast carcinoma and creatine kinase in lung carcinoma, were used in order to provide treatment option for HER-2 treatment and to identify the primary source of carcinoma (Figure 2). However, in one case, it was found that the pathological examination of the patient had been reported as anaplastic ependymoma in a previous health facility, but breast cancer metastasis was found to be the primary diagnosis following the examination of the material obtained from ISCM excision.

There were patients with $(n=3)$ and without $(n=2)$ preoperative carcinoma diagnosis. It was noted that all of the patients had a history of worsening motor deficits and urinary incontinence. 
Neurological examination of the patients included preoperative paraplegia $(n=2)$ and paraparesis $(n=3)$.

The electronic databases were searched using various keywords, and the obtained data were arranged (Table 2). The search results were as follows:

a) Two clinical articles were found using "intramedullary spinal cord metastasis" ( $n=313$ ) as search term. However, when the full texts were evaluated, they were ex cluded from the study because they were not relevant $(30,31)$.

b) When "intramedullary spinal cord metastasis" AND/ OR "carcinoma metastasis" ( $n=163)$ search terms were used, no clinical article was found. "Intramedullary spinal cord metastasis" AND/OR "renal cell carcinoma" search terms yielded three clinical articles $(17,26,27)$.

c) When "intramedullary spinal cord metastasis" AND/OR "breast cancer" ( $n=42)$ search terms were used, five clinical articles were found $(21,23,28-30)$. Nonetheless, they were excluded as they did not meet the study criteria by Eicker et al. (30).

d) Although the search with "intramedullary spinal cord metastasis" AND/OR "intracranial metastasis" terms resulted in 27 articles, there was no clinical article.

e) When "intramedullary spinal cord metastasis" AND/OR "lung adenocarcinoma" $(n=5)$ search terms were used, it was observed that there were only two clinical articles $(18,25)$.

f) When "intramedullary spinal cord metastasis" AND/ OR "lung cancer" ( $n=99)$ search terms were used, there were 10 clinical articles, but only 8 met the inclusion criteria $(10,14-16,19,20,22,24)$.

While $86.66 \%$ of the investigated cases were males in the literature, it was $80 \%$ in our study. The mean age of the patients was $56.2 \pm 15.88$ years according to data given in the literature, whereas it was $63.2 \pm 8.23$ years in our study. The incidence of metastases in the literature was $52.94 \%$ for lung carcinoma, $23.53 \%$ for breast carcinoma, and $17.65 \%$ for RCC. In our study, these rates were $60 \%, 20 \%$, and $20 \%$, respectively. Although it was found that only $35.29 \%$ of the cases in the literature were operated, all our patients underwent surgery. However, it was not clear whether the same surgical technique was applied in other cases.

While time to ISCM calculated from data obtained from the literature was $25.75 \pm 22.37$ months, it was calculated to be $22.75 \pm 32.96$ months in our study. Although time to death after ISCM was $12.2 \pm 12.17$ months in the literature, it was $7.66 \pm 5.68$ months in our study. It was found that all of the cases included in our study had undergone RT and/or CTX treatment, and this rate was $82.35 \%$ in the literature. It was not known whether RT and/or CTX were applied in the remaining cases.

\section{DISCUSSION}

The number of scientific studies in which independent and different outcomes have been achieved in any given field is rapidly increasing, and information accumulation is occurring. In addition, similar investigations may lead to different results. As a result, it is

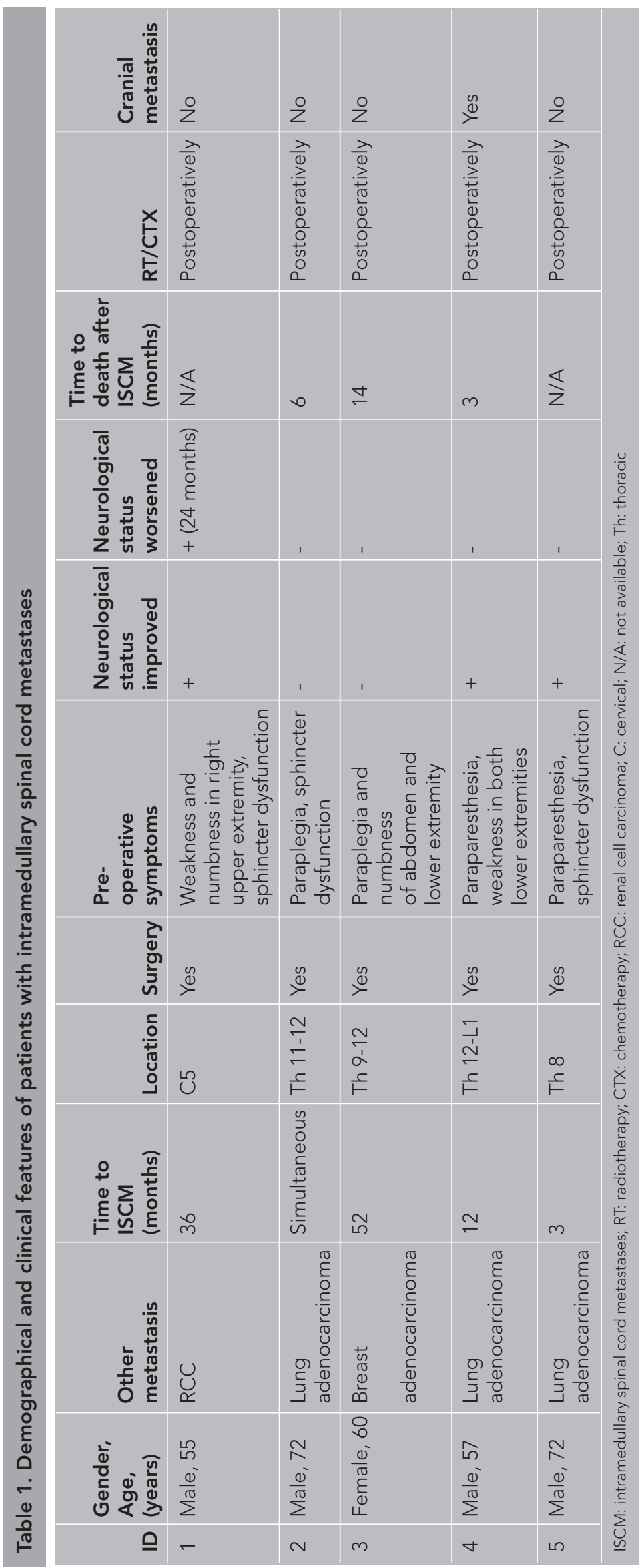



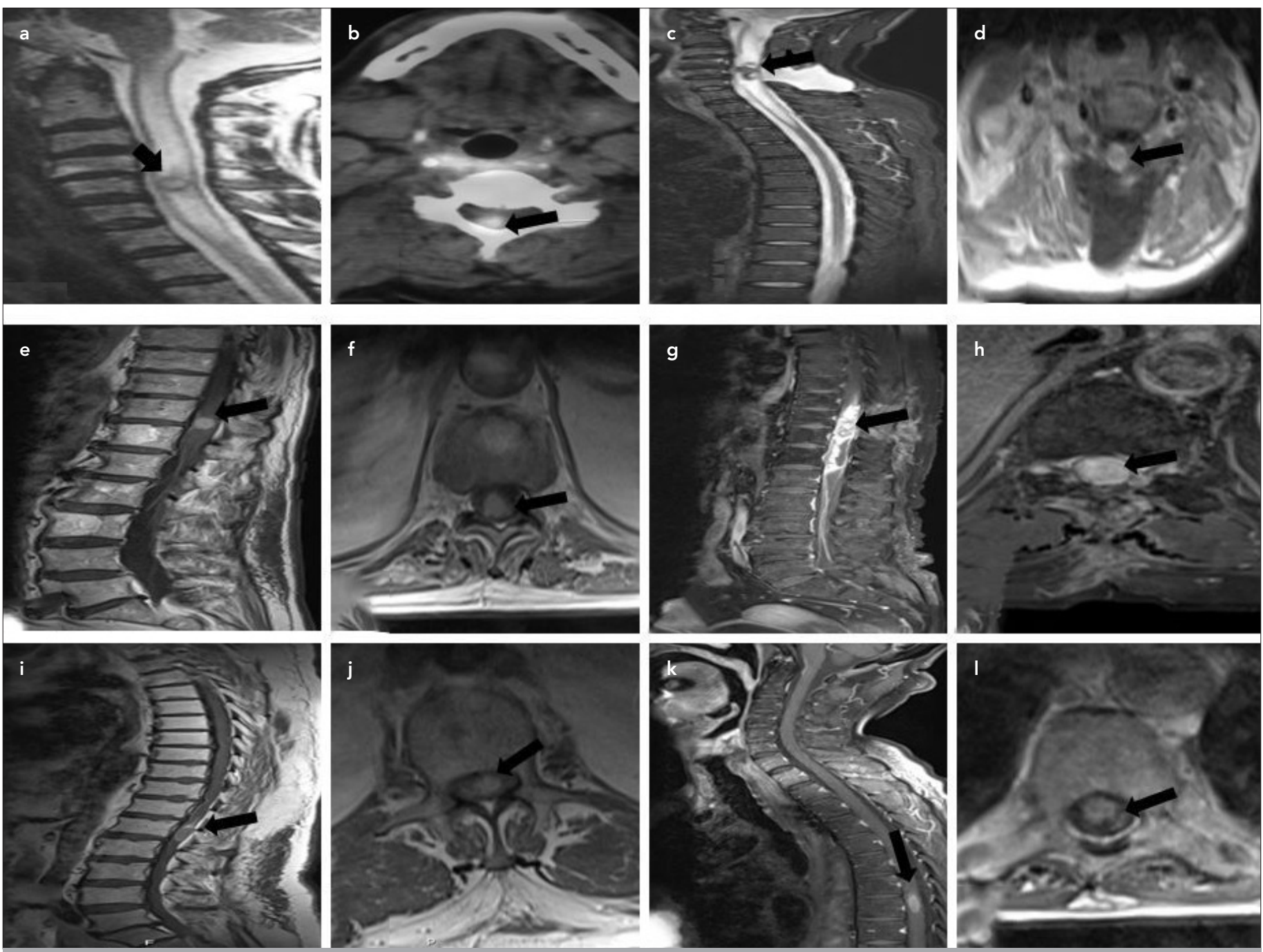

Figure 1. a-I. (a) Pre-operative sagittal T2-weighed cervical MR image of patient 1; (b) Pre-operative axial cervical CT image of patient 1; (c) Postoperative second year contrast-enhanced sagittal cervicothoracic MR image of patient 1 ; (d) Post-operative second year contrast-enhanced axial cervical MR image of patient 1; (e,g,i) Pre-operative contrast-enhanced sagittal thoracolumbar MR image of patient 2,3 and 4; (f,h,j) Pre-operative contrast-enhanced axial thoracolumbar MR image of patient 2,3 and 4; (k,l) Pre-operative contrast-enhanced sagittal and axial cervicothoracic MR image of patient 5

difficult to decide whether the differences may be ignored since the findings of studies are not similar. Owing to such difficulties, different studies on the same topic need to be gathered under a single roof $(32,33)$. The mortality and morbidity of ISCM are high, and it is difficult to determine the optimal treatment approach. The aim of the present study was to collect all findings of ISCM cases under a single roof in light of the literature.

It is known that most ISCMs are often located in the thoracic $(60 \%)$ and lumbosacral (30\%) regions, and the symptoms are related to the primary metastatic area, such as low back pain, sensory deficits, and sphincter dysfunctions $(34,35)$.

In the presence of clinical suspicion in patients with a primary carcinoma, the sensitivity of the whole spinal MRI is quite high, and it is superior to CT/CT myelography. Adjuvant RT and/or CT administration in addition to primary surgery in the treatment of ISM-diagnosed cases is considered almost standard because of the provided improvement in prognosis $(3,5,11,36)$.
Since ISM is rare, primer spinal cord tumors are considered primarily when intramedullary masses are detected after radiological imaging. Although rare, ISMCs can now be seen as cancer diagnosis, and treatment has become widespread. Therefore, when an intramedullary mass is detected in the spinal cord, ISCM should be kept in mind. It should not be forgotten that these patients should undergo metastatic primary tumor focus scanning, and that the excised material might be ISCM.

Among the studies with high levels of evidence, Minomo et al. (10) reported a high-grade lung carcinoma-related ISCM case with short survival. The reported patient had undergone left pneumonectomy 10 months ago, and lung carcinoma-related ISCM was detected following surgery for intramedullary mass at C2 level. They also reported that the patient's neurological symptoms or clinical signs improved, and symptoms due to spinal cord compression disappeared following surgery and RT. They underlined that tumor size might decrease with mul- 

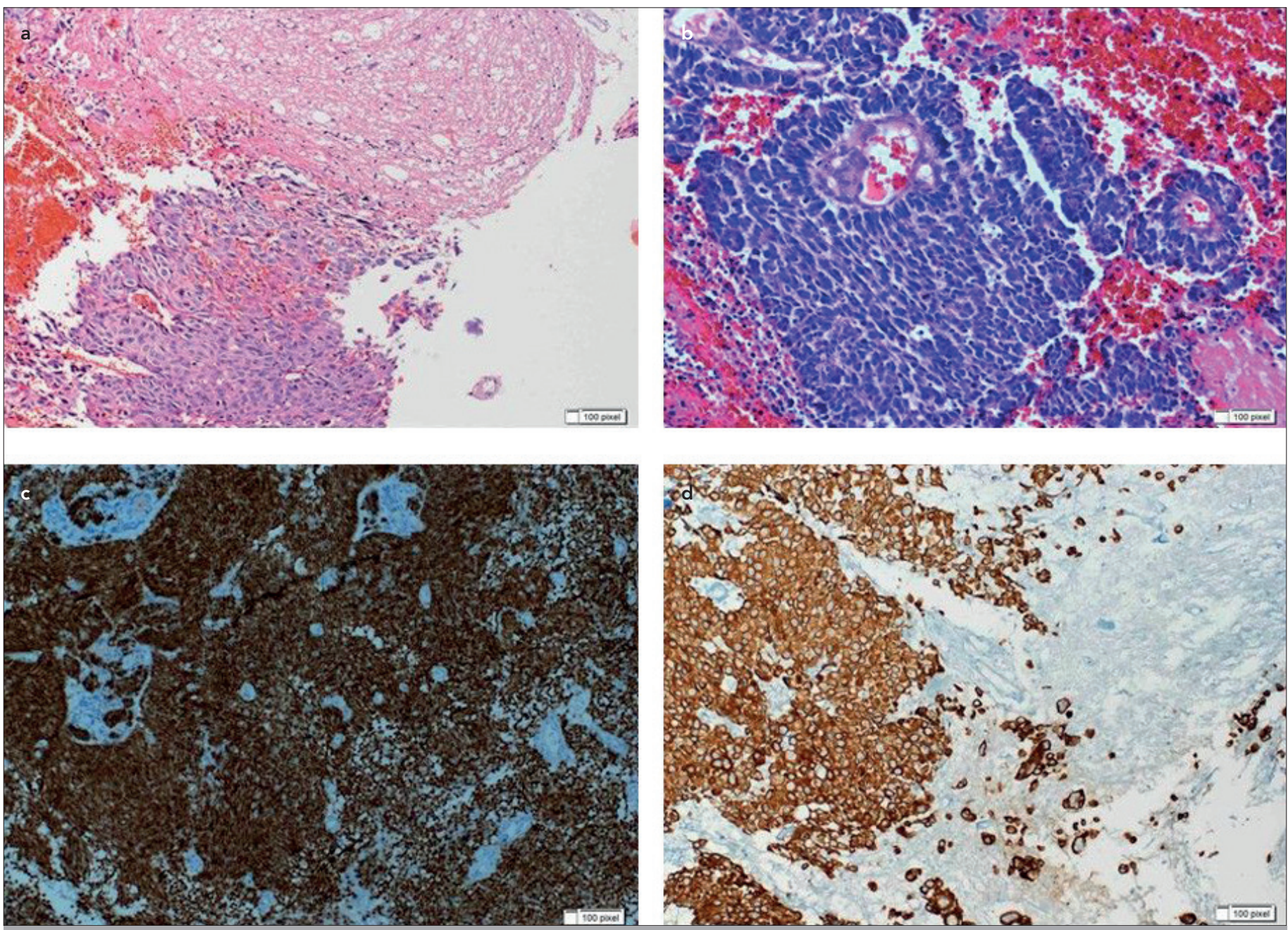

Figure 2. a-d. (a) H \& E stained slide of patient 2 showing predominant mitotic activation and neural parenchyma invasion (10x); (b) $H$ \& $E$ stained slide of patient 3 showing formation of adenoid secretory glands (20x); (c) Pathological examination of immunohistochemically Thyroid Transcription Factor-1 (TTF-1) stained slide of patient 4; (d) Pathological examination of immunohistochemically lung adenocarcinoma-specific CK-7 stained slide of patient 5

timodal local treatment, and that the patient could survive up to 25 months after ISCM diagnosis (10).

In another study conducted by Gao et al. (27), they described a case of RCC-related ISCM with worsening neurological examination 6 years after nephrectomy. In addition, they underlined that ISCM might be observed during the late period.

In another similar study with high level of evidence, Choi et al. (23) reported a metastatic breast carcinoma with late period multilevel ISCM. In their study, they surgically resected two ISCM at C6 and Th12-L1 levels, and the patient underwent multimodal RT and CTX treatment protocols. They emphasized the importance of the absence of recurrence within postoperative 24 months in the presence of clinical improvement. It was found that RCC-related ISCM case was treated 36 months after primary disease diagnosis, and breast carcinoma case was treated 54 months after following emergence of clinical symptoms. This concluded that it might be due to increased survival in patients with malignancy with oncological therapies.
Duransoy et al. (24) and Ozturker et al. (29) both reported one patient who had no prior diagnosis of malignity and then who were diagnosed with ISCM following emergence of clinical signs of ISCM. The reasons for hospital admission included loss of strength in the lower extremities, and imaging studies revealed intramedullary masses at Th9-10 and C2-3 levels $(24,29)$. They postoperatively found that the excised masses were ISCMs originated from lung and breast carcinomas $(24,29)$. In the present study, it was understood that one patient was admitted to the outpatient clinic with clinical findings of ISCM. It is known that such high-grade carcinomas can metastasize systemically much more quickly. Therefore, this indicates that ISCM may present as the initial sign.

Similarly, in two other studies $(18,21)$, ISCM cases with brain metastasis were evaluated. However, these patients were reported to die 4 months after receiving ISCM diagnosis despite multimodal treatment. They stated that ISCM might be a result of possible leptomeningeal spread in patients with brain metastasis. One of the patients in this study was found to have been 


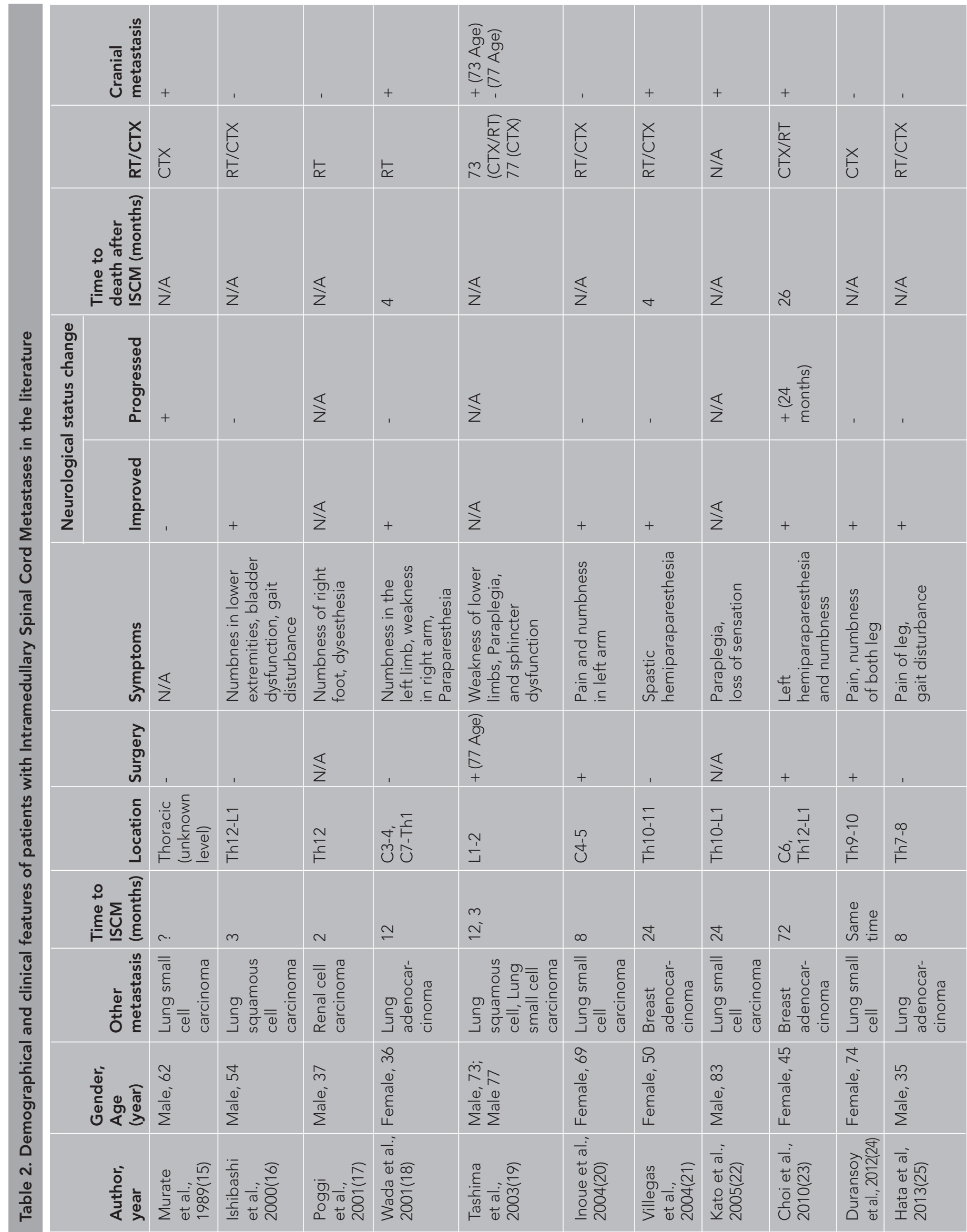


previously operated due to cranial metastasis. However, it was understood that the patient died despite the rapid application of all the current treatment modalities.

Duransoy et al. (24), Gao et al. (27), Inoue et al. (20), Minomo et al. (10), and Park et al. (26) reported a good recovery period after early surgery, especially in the early stages. In this present retrospective study, a significant improvement was reported in the patient with RCC-related ISCM. However, it was found that the deterioration could be stopped if the ISCM had originated from the lung or breast carcinoma. No evidence was found regarding marked improvement in two cases who had undergone surgery probably in the late period, after the development of plegia. This suggests that surgery is the most important treatment in order to stop neurological deterioration or improve neurological status in patients with lung carcinoma-related ISCM with early diagnosis. In addition, it ascertains the significance of questioning the necessity of surgery after the development of plegia.

It has been underlined in the literature that data obtained from analysis results of such different studies should be converted to the same effect size unit. It has been mentioned that a large number of different effect size calculations can be made in the process of transforming research results to be included in such studies into a common measure. What is important when achieving this has been reported to be the calculation of the effect size, which is appropriate to the qualification of the research results to be included in the analysis $(32,33,37)$. Moreover, it is stated that "it should be tested whether the effect sizes are uniformly distributed" while deciding which model to use during analyses. As a result, it has been reported that if the effect sizes are homogeneous, the fixed-effect model can be used, and if not, then the random-effect model should be used $(32,33,38)$. However, as a result of examining the articles that met our criteria for inclusion in our research, there were 16 articles related to the subject. Nonetheless, we have not found any similar study in the literature with data similar to ours. Homogeneity and heterogeneity tests could not be applied. In addition, the assumption that there is only one underlying actual effect that can be predicted from the findings of different studies of this subject could not be statistically argued through meta-analysis. Furthermore, graphical drawing of the findings, such as sample size or reciprocal of variance, could not be performed; instead, they were evaluated by descriptive statistics.

The limitations of our study are as follows: (1) we might have been misled when identifying or evaluating data obtained from articles after the literature review, (2) we might have made mistakes in the evaluation stages of the studies, and (3) we might have been affected from publication bias.

In conclusion, ISCMs are rare, and successful treatment options have increased because of developments in both oncologic diagnosis and surgical treatment techniques. Mortality and morbidity rates can be reduced by evaluation of such cases not only by orthopedic surgeons and neurosurgeons but also by a multidisciplinary way. The prognosis is determined by cell type, location, growth rate, and neurological deterio- 
ration based on carcinoma type. The main purpose of treatment in these patients is spinal cord decompression, and early surgery has a positive effect on preventing and decreasing morbidity and delaying mortality.

\section{CONCLUSION}

Today, surgery is the most effective treatment modality in ISCM. Thus, it is important to remember that patients can be diagnosed correctly and on time, and mortality and morbidity can be significantly reduced through successful outcomes. In such cases, the importance of surgery is questioned once again, especially after the development of plegia. There is an urgent need for prospective studies that provide comparative results of data from multicenter trials involving more cases from both genders and all races in the literature.

Ethics Committee Approval: Authors declared that the research was conducted according to the principles of the World Medical Association Declaration of Helsinki "Ethical Principles for Medical Research Involving Human Subjects", (amended in October 2013).

Informed Consent: Written informed consent was obtained from patients who participated in this study.

Peer-review: Externally peer-reviewed.

Author Contributions: Concept - Ç.Ö.; Design - Y.A.; Supervision -Ç.Ö.; Resources - Y.A.; Materials - N.K.; Data Collection and/or Processing Y.A., N.K.; Analysis and/or Interpretation - Y.A.; Literature Search - Y.A.; Writing Manuscript - Y.A.; Critical Review - Ç.Ö.

Conflict of Interest: The authors have no conflict of interest to declare.

Financial Disclosure: The authors declared that this study has received no financial support.

\section{REFERENCES}

1. Grasso G, Meli F, Patti R, Giambartino F, Florena AM, lacopino DG. Intramedullary spinal cord tumor presenting as the initial manifestation of metastatic colon cancer: case report and review of the literature. Spinal Cord 2007; 45: 793-6. [CrossRef]

2. Mut M, Schiff D, Shaffrey ME. Metastasis to nervous system: spinal epidural and intramedullary metastases. J Neurooncol 2005; 75: 4356. [CrossRef]

3. Jacobs WB, Perrin RG. Evaluation and treatment of spinal metastases: an overview. Neurosurg Focus 2001; 11: e10. [CrossRef]

4. Diehn FE, Rykken JB, Wald JT, Wood CP, Eckel LJ, Hunt CH, et al. Intramedullary spinal cord metastases: prognostic value of MRI and clinical features from a 13-year institutional case series. AJNR Am J Neuroradiol 2015; 36: 587-93. [CrossRef]

5. Lee EQ. Nervous system metastases from systemic cancer. Continuum 2015; 21: 415-28. [CrossRef]

6. Schiff D, O'Neill BP. Intramedullary spinal cord metastases: clinical features and treatment outcome. J Neurooncol 1996; 47: 906-12. [CrossRef]

7. Dunne JW, Harper CG, Pamphlett R. Intramedullary spinal cord metastases: a clinical and pathological study of nine cases. $\mathrm{O} \mathrm{J}$ Med 1986; 61: 1003-20.

8. Fakih M, Schiff D, Erlich R, Logan TF. Intramedullary spinal cord metastasis (ISCM) in renal cell carcinoma: a series of six cases. Ann Oncol 2001; 12: 1173-7. [CrossRef]

9. Asad S, Sher I, Peters-Willke J, Jessup P. Neurotropic cutaneous malignant melanoma with contiguous spread to spinal cord, an extremely rare presentation. J Spine Surg 2016; 2: 76-81. [CrossRef]
10. Minomo S, Tokoro A, Utsumi T, Ishihara M, Akira M, Atagi S. A case of long-term survival after multimodal local treatments of intramedullary spinal cord metastasis of squamous cell lung cancer. J Thorac Dis 2016; 8: E681-3. [CrossRef]

11. Hashii H, Mizumoto M, Kanemoto A, Harada H, Asakura H, Hashimoto $T$, et al. Radiotherapy for patients with symptomatic intramedullary spinal cord metastasis. J Radiat Res 2011; 52: 641-5. [CrossRef]

12. Ding D, Fullard M, Jarrell HS, Jones DE. Intramedullary spinal cord metastasis from salivary ductal carcinoma of the parotid gland mimicking transverse myelitis in a patient with radiologically isolated syndrome. J Neurol Sci 2014; 336: 265-8. [CrossRef]

13. Lijmer JG, Mol BW, Heisterkamp S, Bonsel GJ, Prins MH, van der Meulen JH, et al. Empirical evidence of design-related bias in studies of diagnostic tests. Jama 1999; 282: 1061-6. [CrossRef]

14. Nishio S, Fukui M, Sato Y, Araki K. Intramedullary spinal cord metastasis from lung cancer--a case report (author's transl). Neurol Surg Tokyo 1981; 9: 969-73.

15. Murate T, Hasegawa T, Hasegawa Y, Shimokata K. A case report of small cell lung cancer with extrahepatic biliary obstruction and intramedullary spinal cord metastasis. Nihon Kyobu Shikkan Gakkai Zasshi 1989; 27: 513-7.

16. Ishibashi $Y$, Tokuchi $Y$, Kamachi M, Harada M, Isobe $H$. A case of non-small-cell lung cancer with intramedullary spinal cord metastasis diagnosed pre-mortem. Nihon Kokyuki Gakkai Zasshi 2000; 38: 943-6.

17. Poggi MM, Patronas N, Buttman JA, Hewitt SM, Fuller B. Intramedullary spinal cord metastasis from renal cell carcinoma: detection by positron emission tomography. Clin Nucl Med 2001; 26: 837-9. [CrossRef]

18. Wada H, leki R, Ota T, Iguchi M, Yuasa K, Okamura K, et al. Intramedullary spinal cord metastasis of lung adenocarcinoma causing Brown-Sequard Syndrome. Nihon Kokyuki Gakkai Zasshi 2001; 39: 590-4.

19. Tashima M, Ono N, Noguchi T, Ishikawa H, Kamakari K, Terada Y. Two cases of intramedullary spinal cord metastasis of lung cancer detected with MRI. Nihon Kokyuki Gakkai Zasshi 2003; 41: 320-3.

20. Inoue T, Tanaka E, Sakuramoto M, Minakuchi M, Maeda Y, Maniwa $K$, et al. A case of intramedullary spinal cord metastasis due to small cell lung cancer. Nihon Kokyuki Gakkai Zasshi 2004; 42: 1014-8.

21. Villegas $A E$, Guthrie $T H$. Intramedullary spinal cord metastasis in breast cancer: clinical features, diagnosis, and therapeutic consideration. Breast J 2004; 10: 532-5. [CrossRef]

22. Kato A, Katayama H, Hamada H, Nagao T, Kadowaki T, Ito R, et al. A case of small cell lung cancer with intramedullary spinal cord metastasis. Nihon Ronen Igakkai Zasshi 2005; 42: 567-70. [CrossRef]

23. Choi HC, Yoon DH, Kim SC, Cho KH, Kim SH. Two separate episodes of intramedullary spinal cord metastasis in a single patient with breast cancer. J Korean Neurosurg Soc 2010; 48: 162-5. [CrossRef]

24. Duransoy YK, Mete M, Selcuki M, Isısag I. Isolated solitary intramedullary spinal cord metastasis presenting as the first manifestation of small-cell lung cancer: report of a rare case. Case Rep Neurol Med 2012; 2012: 617280. [CrossRef]

25. Hata Y, Takai Y, Takahashi H, Takagi K, Isobe K, Hasegawa C, et al. Complete response of 7 years' duration after chemoradiotherapy followed by gefitinib in a patient with intramedullary spinal cord metastasis from lung adenocarcinoma. J Thorac Dis 2013; 5: E65-7.

26. Park J, Chung SW, Kim KT, Cho DC, Hwang JH, Sung JK, Lee D. Intramedullary spinal cord metastasis in renal cell carcinoma: a case report of the surgical experience. J Korean Neurosurg Soc 2013; 28: 1253-6. [CrossRef]

27. Gao J, Li Y, Yang Z, Wang R. Intramedullary spinal cord metastasis of renal cell carcinoma 6 years following the nephrectomy. Turk Neurosurg 2014; 24: 294-6.

28. Kawamoto T, Yamashita T, Kaito S, Miura Y. Intramedullary Spinal Cord Metastasis from Breast Cancer Mimicking Delayed Radiation 
Myelopathy: Detection With (18)F-FDG PET/CT. Nucl Med Mol Imaging 2016; 50: 169-70. [CrossRef]

29. Ozturker C, Sivrioglu AK, Sildiroglu HO, Ozturk E, Sonmez G. Breast cancer presenting with intramedullary cervical spinal cord metastasis. Spine J 2016; 16: e463-4. [CrossRef]

30. Eicker SO, Floeth FW, Kamp M, Steiger HJ, Hanggi D. The impact of fluorescence guidance on spinal intradural tumour surgery. Eur Spine J 2013; 22: 1394-401. [CrossRef]

31. Chamoun RB, Alaraj AM, Al Kutoubi, Abboud MR, Haddad GF. Role of temozolomide in spinal cord low grade astrocytomas: results in two paediatric patients. Acta Neurochir 2006; 148: 175-9. [CrossRef]

32. Yilmaz I, Akkaya $S$, Isyar M. Is there a treatment protocol in which platelet-rich plasma is effective? J Orthop 2016; 13: 316-21. [CrossRef]

33. Ali Gumustas S, Isyar M, Topuk S, Yilmaz I, Oznam K, Onay T, et al Systematic Evaluation of Drug-Loaded Hydrogels for Application in Osteosarcoma Treatment. Curr Pharm Biotechnol 2016; 17: 866-72. [CrossRef]
34. Garcia R, Sallabanda K, Santa-Olalla I, Lopez Guerra JL, Aviles L, Sallabanda M,et al. Robotic Radiosurgery for the Treatment of Intramedullary Spinal Cord Metastases: A Case Report and Literature Review. Cureus 2016; 8: e609. [CrossRef]

35. Schiff D. Spinal cord compression. Neurol Clin 2003; 21: 67-86. [CrossRef]

36. Tripathy SR, Deo RC, Mishra S, Dhir MK, Nath PC, Satapathy MC. Intramedullary spinal cord metastasis arising from papillary thyroid carcinoma: A case report and review of literature. Surg Neurol Int 2016; 7(Suppl 13): S375-9. [CrossRef]

37. Robert M, Bernard PCA, Borokhovski YLE, Wade A, Wozney L, Wallet PA, et al. How Does Distance Education Compare With Classroom Instruction? A Meta-Analysis of the Empirical Literature. Rev Educ Res 2004; 74: 379-439. [CrossRef]

38. Berman NG, Parker RA. Meta-analysis: neither quick nor easy. BMC Med Res Methodol 2002; 2: 10. [CrossRef] 